\title{
Overexpression of ThMYB8 mediates salt stress tolerance by directly activating stress-responsive gene expression
}

\author{
zhongyaun liu ${ }^{1}$, tengqian zhang ${ }^{1}$, xinping li $^{1}$, peilong wang ${ }^{1}$, yuanyuan wang $^{1}$, and Caiqiu \\ $\mathrm{GaO}^{1}$
}

${ }^{1}$ Northeast Forestry University

May 5, 2020

\begin{abstract}
MYB transcription factors are important in abiotic stress responses; however, the detailed mechanisms are unclear. Tamarix hispida contains multiple MYB genes. The present study characterized T. hispida MYB (ThMYB8) during salt stress using transgenic T. hispida and Arabidopsis assays. ThMYB8 overexpression and ThMYB8 RNAi analysis demonstrated that ThMYB8 enhanced the salt stress tolerance. Transgenic Arabidopsis overexpressing ThMYB8 displayed significantly increased root growth, fresh weight, and seed germination rate compared with that of the wild-type. Physiological parameters analysis in T. hispida and Arabidopsis showed that ThMYB8 overexpressing plants had the lowest levels of O2-, H2O2, cell death, malondialdehyde, and electrolyte leakage. Overexpression of ThMYB8 regulated $\mathrm{Na}+$ and $\mathrm{K}+$ concentrations in plant tissues while maintaining $\mathrm{K}+/ \mathrm{Na}+$ homeostasis. Analysis using qRT-PCR and ChIP-PCR identified possible downstream ThMYB8regulated genes. ThMYB8 regulated the expression of ThCYP450-2 (cytochrome p450-2), Thltk (leucine-rich repeat transmembrane protein kinase), and ThTIP (aquaporin TIP) by binding to the MBSI motif ('CAACTG') in their promoters. The results indicated that ThMYB8 enhanced salt stress tolerance in $\mathrm{T}$. hispida by regulating gene expression related to the activation of stress-associated physiological changes, such as enhanced reactive oxygen species scavenging capability, maintaining $\mathrm{K}+/ \mathrm{Na}+$ homeostasis, and decreasing the malondialdehyde content and lipid peroxidation cell membranes.
\end{abstract}

\section{Hosted file}

Manuscript . doc available at https ://authorea.com/users/303423/articles/433551-overexpressionof-thmyb8-mediates-salt-stress-tolerance-by-directly-activating-stress-responsive-geneexpression

\section{Hosted file}

Fig.1-8.pdf available at https://authorea.com/users/303423/articles/433551-overexpressionof-thmyb8-mediates-salt-stress-tolerance-by-directly-activating-stress-responsive-geneexpression 